\title{
Tumor Complete Metabolic Response by PET
}

National Cancer Institute

\section{Source}

National Cancer Institute. Tumor Complete Metabolic Response by PET. NCI Thesaurus. Code C116095.

A finding obtained using FDG-PET imaging demonstrating the disappearance of metabolic tumor activity in target and non-target lesions, marked by a decrease in tumor standardized uptake value to the level of surrounding normal tissue. 